\title{
Efficiency of Weed Control Methods and Planting Population on Controlling Weeds and the Economic Feasibility of Onion Productivity Geries, L. S. M. ${ }^{1}$ and Azza E. Khaffagy ${ }^{2}$ \\ ${ }^{1}$ Onion Res. Dept., Field Crops Res. Inst., Agric. Res. Center, Giza, Egypt \\ ${ }^{2}$ Weed Res. Laboratory, Field Crops Res. Inst., Agric. Res. Center, Giza, Egypt
}

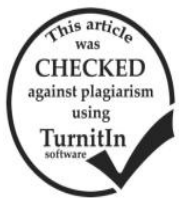

\section{ABSTRACT}

Two field experiments were carried out during two successive winter seasons (2016/17-2017/18) at Agricultural Research Station, Kafr El-Sheikh Governorate, Agricultural Research Center, to study the effect of twenty four treatments which were a combination of eight weed control treatments (i.e., Against, Against with hand hoeing once, Floro, Floro with hand hoeing once, Stomp extra, Stomp extra with hand hoeing once, hand hoeing twice and unweeded check) and three planting population including 120,180 and 240 thousand plants/fad on weeds control, growth characters, onion production and its quality. All tested weed control treatments significantly increased onion growth characters and yield during the two seasons. Weed control treatment by Floro at rate of 750 $\mathrm{cm}^{3} /$ fad + hand hoeing once at 45 days after transplanting, reduced percentage of dry weight of broad-leaved, grassy and total weeds at 60 and 90 days after transplanting by $(97.08 \& 96.39 \%),(90.50 \& 90.50 \%)$ and $(96.20 \& 95.20 \%)$ in the average survey in $2016 / 17$ and 2017/18 seasons, respectively, compared to unweeded check. The same treatment produced the highest average bulb weight, marketable and total bulbs yield per fad by about 45.67, 74.56 and $59.78 \%$ when compared with unweeded check in the average of both seasons, respectively with best storability along with bulbs quality at low cost of onion production, demonstrate that weed control is very important in the onion fields. Also, onion plant grown at 180 thousand plants/fad revealed a very promising effect for marketable and total yield with the average of 17.89 and $20.24 \%$ in conjunction with a great reduction in the total weed biomass $/ \mathrm{m}^{2}$ at 60 and 90 days after transplanting by about 25.51 and $25.77 \%$ in the two seasons compared to 120 thousand plants/fad., respectively. The maximum values of total soluble solids, percentage of dry matter in bulbs, and remaining marketable bulbs after storage for six months were achieved under the density of 120 or 180 thousand plants/fad in both seasons. Total bulb yield/fad showed highly significant negative correlation with each of dry weight of broad-leaved, grassy and total weeds as well as culls yield/fad. Therefore, the use of integration between Floro EC 24\% as post-emergence herbicide at rate of $750 \mathrm{~cm}^{3} / \mathrm{fad}$ (21 days after transplanting) followed by hand hoeing once at 45 days after transplanting plus plant density of 180 thousand plants/fad is the best choice for the onion farmers of this area to achieve maximum onion bulb yield/fad, storability and quality of onions with higher economic returns.

Keywords: Onion, bulb yield, pendimethalin, oxfluorfen, acetochlor and herbicides.

\section{INTRODUCTION}

Onion (Allium cepa, L.) is one of the most important crops, in Egypt. As a top agricultural country in the region and Africa, Egypt ranks high among highly producing countries worldwide with total cultivated area of 162,833 faddan in 2016 producing 2 million tons with an average of 14.43 ton/faddan (Yearly Book of Economics and Statistics of the Agric. Ministry in Egypt, 2017).

One of the strong potential causes of the great reduction in onion yield is related to weed competition, because of the peculiar canopy structure of the onions. The traditional methods of weed control (hoeing and hand weeding) are laborious, expensive and scanty. Furthermore, weeding during serious growth stages is very hard due to increased cost of human works and its rare availability. Further, the use of single herbicides alone for weed control is not quit enough due to its narrow spectrum especially through the long competition period for weeds to this crop.

Some researchers estimated onion yield losses due to weed competition by 40 to $80 \%$ (Channapagoudar and Biradar, 2007) and as high among 70 to $84 \%$ (Swaify, 2009). In this situation the use of broad spectrum weed control herbicides including pre or post emergence herbicides as a single or in combination are good choice to achieve maximum yield with lower cost. Several herbicides (e.g., pendimethalin and oxyflurofen) were used successfully in eliminating onion's weeds. Yet, using such herbicides in combination with hand weeding treatment was more promising option at all levels of enhancing crop

\begin{tabular}{|c|c|c|c|c|c|c|c|c|c|}
\hline \multirow{2}{*}{ Season } & \multirow{2}{*}{$\begin{array}{l}\text { Soil } \\
\text { pH }\end{array}$} & Sand & Silt & Clay & Organic matter & \multirow{2}{*}{$\begin{array}{l}\text { Textural } \\
\text { classes }\end{array}$} & \multirow{2}{*}{\multicolumn{3}{|c|}{$\begin{array}{c}\mathbf{P} \\
\text { Ppm }\end{array}$}} \\
\hline & & & & $\%$ & & & & & \\
\hline $2016 / 17$ & 7.90 & 19.00 & 33.57 & 47.43 & 1.81 & Clayey & 27.15 & 16.90 & 280.0 \\
\hline $2017 / 18$ & 7.88 & 19.27 & 30.33 & 50.40 & 1.73 & Clayey & 22.37 & 18.45 & 277.10 \\
\hline
\end{tabular}

Previous summer crop was cotton in first season and maize in second one. The recommended rates of NPK

productivity (Jilani et al., 2007, Hussain et al., 2008, Kalhapure and Shete, 2012 and Kalhapure et al., 2014).

Also adaptation can integrate partially for weed management in this poor competition crop. Many authors reported that wider spacing caused higher yield per plant, although the closer spacing gave higher yield per unit area due to increased plant density up to a certain limit (Abdelmasieh, 2017 and Walle et al., 2018) and the use optimum of plant population or spacing has double characteristic, avoiding strong competition between plants for growth factors such as nutrients, water and light.

Thus, the objective of this study was to evaluate the efficiency of several herbicides in comparison with manual weeding under three planting density and its possible integration for weed control during the competition duration period, select the most effective herbicide in weed control in onion transplanted and their consequent effects on different parameters of onion crop including yield, yield components and its storage characters.

\section{MATERIALS AND METHODS}

Two field experiments were conducted during two successive winter seasons 2016/17 and 2017/18 at Sakha Agricultural Research Station, Kafr El-Sheikh Governorate, Egypt. Mechanical and chemical properties of soil samples were determined according to Klute (1986) and Jackson (1973), which taken up to $30 \mathrm{~cm}$ depth from the experimental sites before transplanting (Table 1) 
applied, respectively. All phosphorus and potash rates were applied at the time of soil bed preparation, half of the amount nitrogen was applied after one month of transplanting and half was applied at 2 months after transplantation. Irrigation and insect/disease control measures were performed as recommended by Ministry of Agriculture during the two seasons of study.

The plot size was $3 \times 3.5 \mathrm{~m}$ with five ridges $60 \mathrm{~cm}$ apart between ridges, ridging directions was north-south, transplanting was done on both sides of the ridge. Herbicides were sprayed in both field experiments by CP3 backpack sprayer with 200 liters water volume per fad. In both seasons, Giza red seeds were planted in the nursery on $15^{\text {th }}$ and $16^{\text {th }}$ October and plants were transplanted in the field after 60 days on mid of December.

Weed control and plant population treatments are as following:-

$\mathrm{T}_{1}$ - Against EC 43\% (acetochlor $37.5 \%+$ oxfluorfen $5.5 \%) 2$ - chloro - $N$ - (ethoxymethyl) - N - (2- ethyl 6-methylphenyl) acetamide + 2-chloro-1-(3-ethoxy-4nitrophenoxy)-4-(trifluoromethyl) benzene, at rate of $1.25 \mathrm{~L} / \mathrm{fad}$, soil surface application directly, (before irrigation and transplanting).

$\mathrm{T}_{2}$ - Against EC $43 \%$, followed by one hand hoeing at 45 days after transplanting (DAT).

$\mathrm{T}_{3}$ - Floro EC 24\% (oxfluorfen) 2-chloro-1-(3-ethoxy-4nitrophenoxy)-4-(trifluoromethyl) benzene, at rate of $750 \mathrm{~cm}^{3} / \mathrm{fad}$, application directly, (after 21 days transplanting).

$\mathrm{T}_{4^{-}}$Floro EC $24 \%$, followed by one hand hoeing at 45 DAT.

$\mathrm{T}_{5}$-Stomp extra CS 45.5\% (pendimethalin) [N-(1ethylpropyl)-3,4 diethyl- 2,6-dinitrobenzenamine], at the rate of $1.5 \mathrm{~L} / \mathrm{fad}$, soil surface application directly, (before irrigation and transplanting).

$\mathrm{T}_{6}$ - Stomp extra CS $45.5 \%$, followed by one hand hoeing at 45 DAT.

$\mathrm{T}_{7}$ - Hand hoeing twice done, 30 and 45 DAT.

$\mathrm{T}_{8}$ - Unweeded check.

Meanwhile planting population was allocated in vertical direction:

$\mathrm{B}_{1}-30$ plants $/ \mathrm{m}^{2}$ (120 thousand plants/fad).

$\mathrm{B}_{2}-45$ plants $/ \mathrm{m}^{2}$ (180 thousand plants/fad).

$\mathrm{B}_{3}-60$ plants $/ \mathrm{m}^{2}$ (240 thousand plants/fad).

Data recorded:

\section{A. Dry weight of annual weeds:}

Annual weeds were hand pulled after 60 and 90 DAT identified to species and classified into three categories (broad-leaved, grassy and total weeds). The weight of the dry grass was determined $\left(\mathrm{g} / \mathrm{m}^{2}\right)$, after drying in a forced draft oven at $70 \mathrm{C}^{\circ}$ for 48 hours.

\section{B. Weed species susceptibility rating of weeds:}

The susceptibility of weeds to herbicides was measured after 25 days from application the herbicides by the reduction percentage of the dry weight of each species compared to the un-weeded check according to Frans and Talbert (1977) as follow:

- Susceptible $\quad(\mathrm{S})=>90 \%$.

- Moderately susceptible (MS) $=80-89 \%$.

- Moderately tolerant $\quad(\mathrm{MT})=60-79 \%$.

- Tolerant $\quad(\mathrm{T})=<60 \%$

\section{Vegetative growth characteristics of crop:}

Samples of ten onion plants were collected randomly from each plot after 90 and 120 DAT to evaluate onion growth attributes, i.e., plant height $(\mathrm{cm})$, number of leaves/plant, bulb diameter $(\mathrm{cm})$ and plant dry weight $(\mathrm{g})$.

\section{Onion bulbs yield:}

The onion yield were harvested when $50 \%$ plant tops were down of each plot and after harvest, onion plants were left in the field to cure for two weeks, then tops were removed, and the following data: average weight of the bulb (g), marketable, culls and total yield ( $\mathrm{t} /$ fad) were recorded.

\section{E. Bulb quality:}

At the time of harvest, ten bulbs were randomly taken from each plot and bulb diameter $(\mathrm{cm})$, total soluble solids (T.S.S \%) were analyzed by hand Refractrometer and percentage of dry matter in bulbs (D.M. \%) were estimated.

\section{F. Storability of onion bulbs:}

Storability was measured as percentage of marketable total loss in weight of bulbs during a storage period of six months. Then rotting and sprouting bulbs were discarded and the remaining marketable bulbs were weighted.

G- Economic feasibility study:

The following economic criteria were used to determine the net income (LE) and economic profitability:

1. Total costs of onion production (L.E./ fad): as affected by different treatments.

2. Total income $($ L.E. $/$ fad $)=($ Price L.E. $/$ ton $) \times$ Yield $($ ton $/$ fad $)$. 3. Net farm return $($ L.E./fad $)=$ Total income - Total costs.

4. Benefit/Cost ratio $(\mathrm{B} / \mathrm{C})=$ Total income/ Total cost.

One ton of marketable onion $=2000$ L.E. and one ton of culls onion $=800$ L.E. as an average of the two seasons. The economic return was calculated based on current local market price of onion bulbs and cost of input. Economical evaluation was conducted using the formulas described by Cimmyt, 1988.

\section{Correlation study:}

Simple correlation matrix was carried out for the two seasons to investigate the relationship between dry weight of different weed categories and onion yield and its quality according to Steel and Torrie (1980).

Experimental Design and Statistical Analysis:

Each experiment was designed in a strip-plot in RCBD design with four replications. Twenty four treatments which were a combination of eight weed control treatments in horizontal plots, also, three levels of planting population in vertical plots. The obtained data were statistically analyzed and treatment effects were compared using least significant difference test (LSD, P <0.05) as described by Gomez and Gomez (1984).

\section{RESULTS AND DISCUSSION}

A. Effect of weed control methods and planting population on weeds:

List of most important weeds present in the field:

The common weed species, at the experimental sites, were Phalaris minor, (as annual grassy weeds); Rumex dentatus, Anagallis arvensis, Malva parviflora, Chenopodium album, Coronopus sp, Beta vulgaris, Ammi majus and Melilotus indica (as annual broad-leaved weeds).

Results in Table 2 showed the significant variations among different treatments under investigation. They 
caused a significant reduction in the dry weight of broadleaved, grassy and total annuals weeds at 60 and 90 DAT. Along with Floro at rate of $750 \mathrm{~cm}^{3} / \mathrm{fad}$, application directly 21 days after transplanting followed by hand hoeing once at 45 DAT gave a significant reductions in dry weight of broad-leaved, grassy and total annuals weeds at 60 and 90 DAT by (97.08 \& 96.39\%), (90.50 \& 90.50\%) and $(96.20 \& 95.20 \%)$ in the average survey in $2016 / 17$ and 2017/18 seasons, respectively, compared to unweeded check. On the other hand, results due to successfully destroy most of the weed were much effective in reduce the weed density as the field was invaded by all types of weeds. Hence, weed competition by post emergence herbicide implementation was furthermore enhanced by combination hand hoeing at 45 DAT in weed control treatments, which provides efficient and prolonged weed control and kept the crop weed free during the critical periods of competition. These results are in harmony with Khokhar et al. (2006), Chandrika et al. (2009), Kalhapure et al. (2014) and Elian et al. (2016), Gaharwar et al. (2017) and Ramalingam et al. (2017).

Data presented in Table 2 showed that increasing planting population from 120 to 240 thousand plants/fad decreased dry weight of broad-leaved, grassy and total annual weeds by $34.10,35.70$ and $34.40 \%, 35.40,35.60$ and $35.40 \%$ at 60 and 90 DAT respectively, as an average in both seasons. These were anticipated due to the less inter-specific competition between onion and weeds plants in the lowest density as compared to the high density, which caused decreasing in light transmittance through the leaf canopy of crops, planted in narrow rows or at high populations could suppress growth and development of weeds. These results are in coincidence with those obtained by Abdelmasieh (2017) and Walle et al. (2018).

Table 2. Effect of weed control treatments and planting population on dry weight of broad-leaved, grassy and total weeds $\left(\mathrm{g} / \mathrm{m}^{2}\right)$ after 60 and 90 day from transplant during 2016/17 and 2017/18 winter seasons.

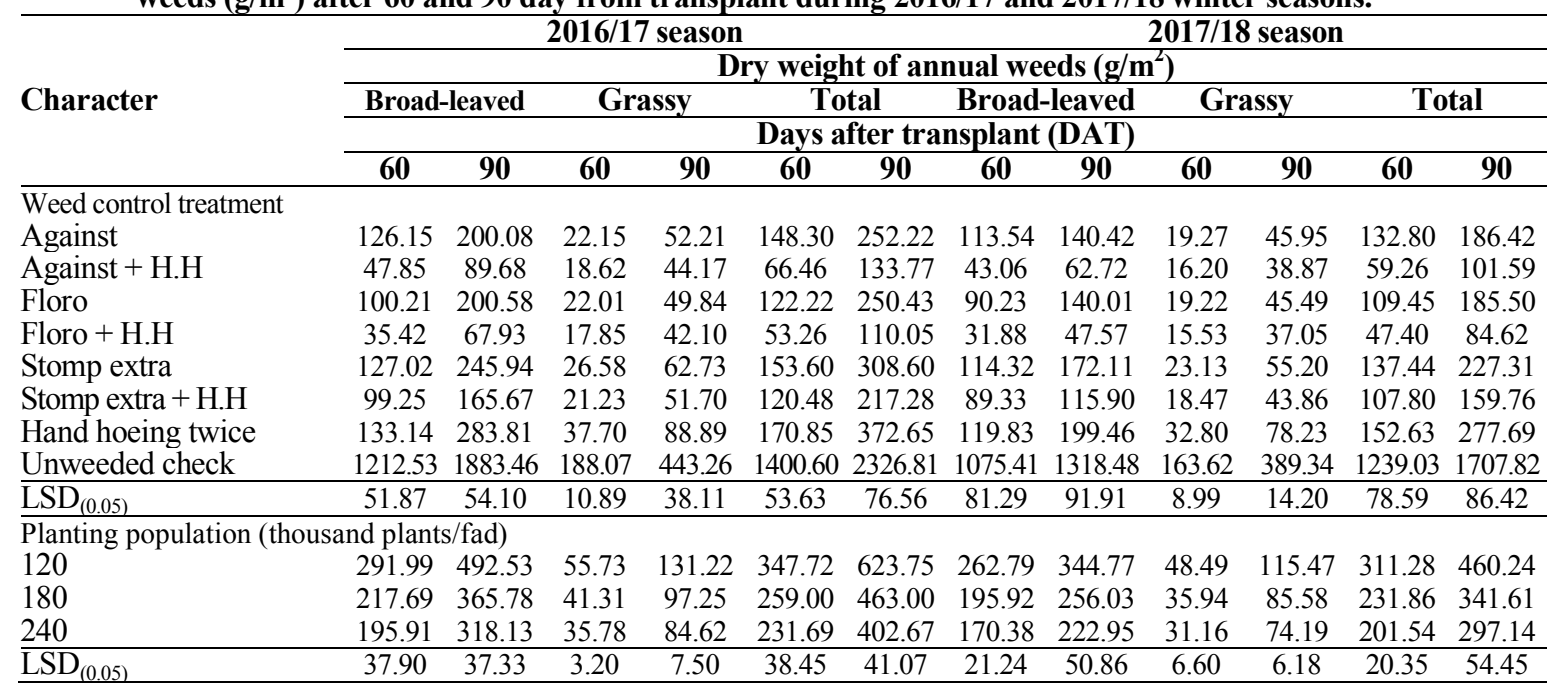

H.H: Hand hoeing

Results in Table 3 indicated that the effect of interactions between weed control treatments and planting population on dry weight of broad-leaved, grassy and total annual weeds were significant at 5\% level. All weed control treatments studied in its combinations with hand hoeing was highly effective for reducing the dry weight of broad-leaved, grass and total annual weeds compared to untreated or using different treatments alone. This means that applying one supplementary hoeing was essential to eliminate the weed plants that survived or escaped from herbicides. Total annual weeds tended to decrease under high densities than under low densities, this may be attributed to the less light transparency, which falls on weeds and consequently weed growth was decreased. Application of Floro at $750 \mathrm{~cm}^{3} / \mathrm{fad}$ plus hand hoeing once at 45 DAT reduced the density of total annual weeds (96.50 to $95.60 \%$ ) at 60 and 90 DAT respectively, the average in both seasons in onion compared with the untreated plots. Several research findings showed that Floro has successfully controlled total annul weeds in onion. The best results were obtained from the interaction between high plant densities ( 240 thousand plants/fad) with weed control treatments followed by 180 thousand plants/fad as compared with low plant density (120 thousand plants/fad) in the same weed control treatments under studying. Similar observations were achieved by Kalhapure et al. (2014).

\section{B. susceptibility rating of weeds:}

Nine weed species and eight herbicides treatments according to the scale of the susceptibility scores of used by Frans and Talbert (1977) were measured depending on the reduction $\%$ of the dry weight of each species in $\mathrm{g} / \mathrm{m}^{2}$ of any herbicide compared with broadleaved and grassy weeds untreated, which measured as mentioned in Table 4, during 2016/17 and 2017/18 seasons, Rumex dentatus, Anagallis arvensis, Malva parviflora, Chenopodium album, Coronopus sp, Beta vulgaris, Ammi majus and Melilotus indica as annual broad-leaved as well Phalaris minor, as annual grassy weeds were susceptible (S) and moderate susceptible (MS) to Floro $\left(750 \mathrm{~cm}^{3} / \mathrm{fad}\right)$ plus once hand hoeing at 45 DAT with ranged between 90$97 \%$, followed by Against plus hand hoeing once percent $89-90 \%$, Stomp extra + hand hoeing once by $85-90 \%$, Floro $750 \mathrm{~cm}^{3} /$ fad by ranged between $85-90 \%$. Herbicides Floro, Against and Stomp extra approximately gave the moderate susceptible (MS) to the previous weeds species meaning that there herbicides had wide spectrum of weed control. The same trend with little differences was observed in weed control treatments in the second season. 
Table 3. The interaction effect between weed control treatments and planting population on dry weight of broadleaved, grassy weeds $\left(\mathrm{g} / \mathrm{m}^{2}\right)$ and total annual weeds at 60 and 90 DAT during 2016/17 and 2017/18 seasons.

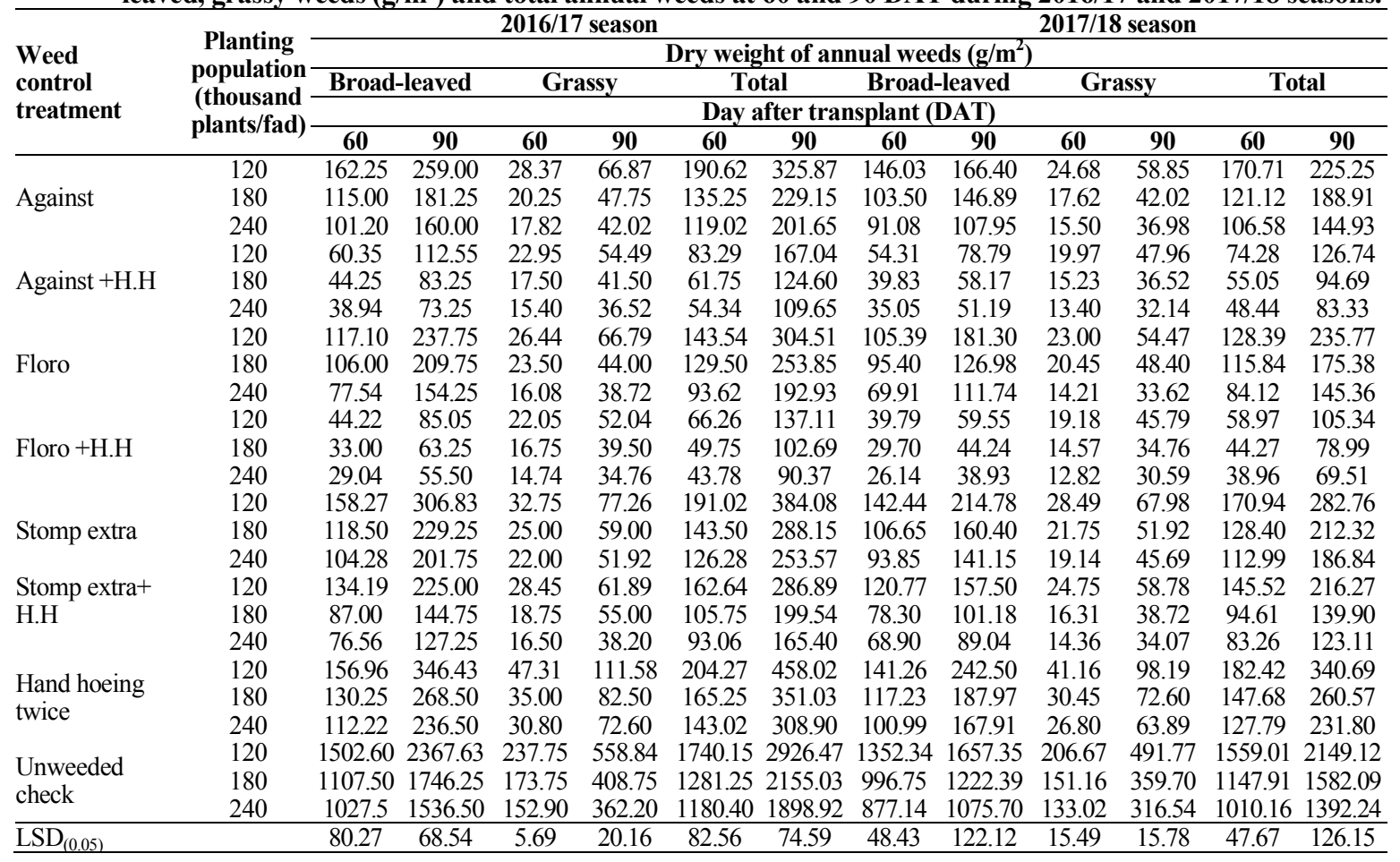

\section{H.H: Hand hoeing}

Table 4. Susceptibility of annual weed species to some herbicides treatments at 60 DAT during $2016 / 17$ and 2017/18 winter seasons.

\begin{tabular}{|c|c|c|c|c|c|c|c|c|c|}
\hline \multirow{3}{*}{$\begin{array}{l}\text { Weeds species } \\
\text { Herbicides }\end{array}$} & \multicolumn{8}{|c|}{ Controlling $\%$ \& weeds species susceptibility to herbicides } & \multirow{2}{*}{$\begin{array}{c}\text { Species of an } \\
\text { annual grassy } \\
\text { weeds }\end{array}$} \\
\hline & \multicolumn{8}{|c|}{ Species of an annual broad-leaved weeds $\left(\mathrm{g} / \mathrm{m}^{2}\right)$} & \\
\hline & $\begin{array}{c}\text { Rumex } \\
\text { dentatus }\end{array}$ & $\begin{array}{c}\text { Anagallis } \\
\text { arvensis }\end{array}$ & $\begin{array}{c}\text { Malva } \\
\text { parviflora }\end{array}$ & $\begin{array}{c}\text { Chenopodium } \\
\text { album }\end{array}$ & $\begin{array}{c}\text { Coronopus } \\
\text { sp } \\
\end{array}$ & Is $\begin{array}{r}\text { Beta } \\
\text { vulgar }\end{array}$ & $\begin{array}{l}\text { Ammi } \\
\text { majus }\end{array}$ & $\begin{array}{c}\text { Melilotus } \\
\text { indica }\end{array}$ & $\begin{array}{c}\text { Phalaris } \\
\text { minor }\end{array}$ \\
\hline & \multicolumn{9}{|c|}{$2016 / 17$ season } \\
\hline Against & $86(\mathrm{MS})$ & $87(\mathrm{MS})$ & 88 (MS) & $86(\mathrm{MS})$ & $87(\mathrm{MS})$ & 89MS) & 86 (MS) & 88(MS) & 87 (MS) \\
\hline Against + H.H & $90(\mathrm{~S})$ & 89 (MS) & $90(\mathrm{~S})$ & $90(\mathrm{~S})$ & 89 (MS) & $94(\mathrm{~S})$ & $91(\mathrm{~S})$ & $91(\mathrm{~S})$ & 90 (MS) \\
\hline Floro & 89 (MS) & 85 (MS) & 89 (MS) & 87 (MS) & 89 (MS) & $90(\mathrm{~S})$ & 89 (MS) & 89(MS & 89 (MS) \\
\hline Floro + H.H & $95(\mathrm{~S})$ & $90(\mathrm{~S})$ & $92(\mathrm{~S})$ & $93(\mathrm{~S})$ & $91(\mathrm{~S})$ & $97(\mathrm{~S})$ & $95(\mathrm{~S})$ & $93(\mathrm{~S})$ & 91 (MS) \\
\hline Stomp extra & 80 (MS) & 84 (MS) & 83 (MS) & 83 (MS) & 83 (MS) & 87MS) & 83 (MS) & 85(MS) & 86 (MS) \\
\hline \multirow[t]{2}{*}{ Stomp extra + H.H } & 89 (MS) & 88 (MS) & $90(\mathrm{~S})$ & 85 (MS) & 88 (MS) & $90(\mathrm{~S})$ & $90(\mathrm{~S})$ & $90(\mathrm{~S})$ & 89 (MS) \\
\hline & \multicolumn{9}{|c|}{$2017 / 18$ season } \\
\hline Against & 87 (MS) & 87 (MS) & 88 (MS) & $86(\mathrm{MS})$ & 88 (MS) 8 & $87 \mathrm{MS}$ & 86 (MS) & 89MS & 88 (MS) \\
\hline Against+ H.H & $90(\mathrm{~S})$ & $91(\mathrm{~S})$ & $90(\mathrm{~S})$ & $90(\mathrm{~S})$ & $90(\mathrm{~S})$ & $91(\mathrm{~S})$ & $91(\mathrm{~S})$ & $92(\mathrm{~S})$ & 91 (MS) \\
\hline Floro & 89 (MS) & 88 (MS) & 89 (MS) & 88 (MS) & $90(\mathrm{~S})$ & 89MS) & 89 (MS) & $90(\mathrm{~S})$ & 89 (MS) \\
\hline Floro +H.H & $93(\mathrm{~S})$ & $92(\mathrm{~S})$ & $92(\mathrm{~S})$ & $91(\mathrm{~S})$ & $92(\mathrm{~S})$ & $95(\mathrm{~S})$ & $92(\mathrm{~S})$ & $94(\mathrm{~S})$ & 93 (MS) \\
\hline Stomp extra & 85 (MS) & 86 (MS) & 83 (MS) & $84(\mathrm{MS})$ & 85 (MS) & 85MS) & 84 (MS) & 87 (MS) & 86 (MS) \\
\hline Stomp extra + H.H & 89 (MS) & 89 (MS) & $90(\mathrm{~S})$ & 89 (MS) & 89 (MS) & $90(\mathrm{~S})$ & $90(\mathrm{~S})$ & $90(\mathrm{~S})$ & $90(\mathrm{~S})$ \\
\hline
\end{tabular}

$\begin{array}{lcccc}\text { Susceptible } & (\mathrm{S})=>90 \% . & \text { Moderately susceptible }(\mathrm{MS})=80-89 \% & \text { H.H: Hand hoeing }\end{array}$

Moderately tolerant $($ MT) $=60-79 \%$ Tolerant $\quad(T)=<60 \%$

\section{Vegetative growth characteristics:}

Statistical analysis of the data showed that different weed control treatments significantly affected the vegetative growth characteristics of onion plants at 90 and 120 DAT during 2016/17 and 2017/18 seasons as compared with the unweeded check treatment (Table 5). Consequently, the highest values of plant height, number of leaves per plant, bulb diameter and plant dry weight were obtained from Floro followed by one hand hoeing once at 45 DAT as compared with all the other herbicidal treatments. Meantime, unweeded check listed the lowest values of plant height, number of leaves/ plant, bulb diameter and plant dry weight of onion plants, followed by hand hoeing twice and Stomp extra alone in both seasons. Similar results of application of oxfluorfen in onion were also reported by Gaharwar et al. (2017), Ramalingam et al. (2017) and kumara et al. (2018).

Concerning the effect of planting population, results in Table 5 reflected that plant density significantly affected vegetative growth characters at the different two samples in both seasons. The highest values of number of leaves/ plant, bulb diameter and plant dry weight of onion bulbs were always associated with the lowest planting population (120 thousand plants/fad) followed by 45 and 60 plants $/ \mathrm{m}^{2}$ (180 and 240 thousand plants/fad, respectively) and this trend was correct in the two seasons. These findings could be attributed to the role of competition among plants on the available resources with increasing plant density. Our results are in agreement with the findings of Bardisi et al. (2013) and Walle et al. (2018). 
Results in Table 6 revealed that the interaction between weed management practices and planting population had significant effects on vegetative growth characters of onion at 90 and 120 DAT in the two seasons. The best results for number of leaves/plant, bulb diameter (cm) and plant dry weight $(\mathrm{g})$ were obtained in weed

Table 5. Plant height $(\mathrm{cm})$, number of leaves per plant, bulb diameter $(\mathrm{cm})$ and plant dry weight $(\mathrm{g})$ of onion plants as affected by different herbicides treatments and planting population at 90 and 120 DAT in 2016/17 and 2017/18 seasons.

\begin{tabular}{|c|c|c|c|c|c|c|c|c|c|c|c|c|c|c|c|c|}
\hline \multirow{4}{*}{ Character } & \multicolumn{8}{|c|}{ 2016/17 season } & \multicolumn{8}{|c|}{ 2017/18 season } \\
\hline & \multicolumn{2}{|c|}{$\begin{array}{l}\text { Plant height } \\
\text { (cm) }\end{array}$} & \multicolumn{2}{|c|}{$\begin{array}{c}\text { No. of } \\
\text { leaves/plant }\end{array}$} & \multicolumn{2}{|c|}{$\begin{array}{c}\text { Bulb } \\
\text { diameter(cm) }\end{array}$} & \multicolumn{2}{|c|}{$\begin{array}{l}\text { Plant dry } \\
\text { weight (g) }\end{array}$} & \multicolumn{2}{|c|}{$\begin{array}{c}\text { Plant height } \\
\text { (cm) }\end{array}$} & \multicolumn{2}{|c|}{$\begin{array}{c}\begin{array}{c}\text { No. of } \\
\text { leaves/plant }\end{array} \\
\end{array}$} & \multicolumn{2}{|c|}{$\begin{array}{c}\text { Bulb } \\
\text { diameter(cm) }\end{array}$} & \multicolumn{2}{|c|}{$\begin{array}{l}\text { Plant dry } \\
\text { weight (g) }\end{array}$} \\
\hline & \multicolumn{16}{|c|}{ Days after transplant (DAT) } \\
\hline & 90 & 120 & 90 & 120 & 90 & 120 & 90 & 120 & 90 & 120 & 90 & 120 & 90 & 120 & 90 & 120 \\
\hline \multicolumn{17}{|c|}{ Weed control treatment } \\
\hline Against & 54.09 & 60.65 & 5.68 & 6.60 & 2.02 & 5.23 & 9.94 & 18.70 & 52.93 & 66.23 & 4.90 & 7.85 & 1.88 & 4.64 & 8.46 & 15.55 \\
\hline Against + H.H & 62.83 & 73.07 & 7.06 & 7.83 & 2.47 & 5.67 & 11.60 & 21.40 & 55.89 & 73.58 & 5.67 & 8.57 & 2.39 & 5.16 & 10.50 & 19.22 \\
\hline Floro & 59.67 & 67.25 & 6.03 & 7.38 & 2.22 & 5.35 & 10.66 & 19.41 & 53.89 & 67.70 & 5.25 & 7.97 & 1.97 & 4.72 & 9.01 & 16.44 \\
\hline Floro + H.H & 67.78 & 78.10 & 7.30 & 8.74 & 2.74 & 6.28 & 12.24 & 22.66 & 58.13 & 75.06 & 6.05 & 8.75 & 2.66 & 5.62 & 11.60 & 20.33 \\
\hline & 52.84 & 63.26 & 5.45 & 6.34 & 1.73 & 5.00 & 9.01 & 17.83 & 51.16 & 63.34 & 4.83 & 7.54 & 1.68 & 4.48 & 8.25 & 14.18 \\
\hline & 60.18 & 70.35 & 6.15 & 7.56 & 2.30 & 5.57 & 11.28 & 20.11 & 55.03 & 70.95 & 5.41 & 8.40 & 2.15 & 4.91 & 9.93 & 17.09 \\
\hline Han & 49.36 & 57.31 & 5.33 & 6.03 & 1.47 & 4.34 & 7.99 & 13.27 & 50.43 & 60.27 & 4.28 & 6.87 & 1.46 & 3.91 & 6.77 & 11.97 \\
\hline Unwee & 41.95 & 52.09 & 4.62 & 5.41 & 1.05 & 3.44 & 5.34 & 5.74 & 47.11 & 53.73 & 3.81 & 5.50 & 0.74 & 3.18 & 5.09 & 8.23 \\
\hline $\mathrm{LSD}_{(0.05)}$ & 2.25 & 2.18 & 0.22 & 0.22 & 0.21 & 0.17 & 0.26 & 0.64 & 1.84 & 2.55 & 0.25 & 0.31 & 0.09 & 0.30 & 0.36 & 0.81 \\
\hline \multicolumn{17}{|c|}{ Planting population (thousand plants/fad) } \\
\hline 120 & 53.52 & 63.41 & 6.05 & 7.24 & 2.21 & 5.47 & 10.85 & 20.28 & 50.36 & 62.99 & 5.33 & 8.48 & 1.99 & 5.27 & 10.53 & 17.34 \\
\hline 180 & 55.96 & 65.21 & 5.96 & 6.91 & 1.99 & 5.08 & 9.76 & 18.37 & 53.20 & 64.44 & 4.90 & 7.52 & 1.87 & 4.63 & 9.04 & 15.02 \\
\hline 240 & 58.78 & 67.17 & 5.84 & 6.80 & 1.80 & 4.78 & 8.66 & 13.51 & 55.65 & 71.64 & 4.85 & 7.05 & 1.74 & 3.83 & 6.53 & 13.76 \\
\hline$\overline{\mathrm{LSD}_{(005)}}$ & 2.35 & 1.92 & 0.10 & 0.08 & 0.19 & 0.15 & 0.11 & 0.51 & 1.44 & 2.66 & 0.21 & 0.26 & 0.10 & 0.22 & 0.29 & 0.38 \\
\hline
\end{tabular}

H.H: Hand hoeing

Table 6. Effect of the interaction between some herbicides treatments and planting population on some onion growth characters at 90 and 120 DAT in 2016/17 and 2017/18 seasons.

\begin{tabular}{|c|c|c|c|c|c|c|c|c|c|c|}
\hline \multirow{4}{*}{$\begin{array}{l}\text { Weed control } \\
\text { treatment }\end{array}$} & \multirow{4}{*}{$\begin{array}{c}\text { Planting } \\
\text { population } \\
\text { (thousand } \\
\text { plants/fad) }\end{array}$} & \multicolumn{6}{|c|}{ 2016/17season } & \multicolumn{3}{|c|}{ 2017/18 season } \\
\hline & & $\begin{array}{c}\text { Plant } \\
\text { height }(\mathrm{cm})\end{array}$ & $\begin{array}{r}\text { No. of } \\
\text { /pla }\end{array}$ & $\begin{array}{l}\text { leaves } \\
\text { int }\end{array}$ & $\begin{array}{c}\text { Bulb } \\
\text { diameter(cm) }\end{array}$ & \multicolumn{2}{|c|}{$\begin{array}{c}\text { Plant dry } \\
\text { weight (g) }\end{array}$} & $\begin{array}{c}\text { Plant } \\
\text { height }(\mathrm{cm})\end{array}$ & $\begin{array}{c}\text { No. of } \\
\text { leaves/plant }\end{array}$ & $\begin{array}{l}\text { Plant dry } \\
\text { weight (g) }\end{array}$ \\
\hline & & \multicolumn{9}{|c|}{ Day after transplant (DAT) } \\
\hline & & 120 & 90 & 120 & 120 & 90 & 120 & 90 & 90 & 90 \\
\hline \multirow{4}{*}{ Against } & 120 & 59.88 & 5.72 & 6.97 & 5.65 & 11.72 & 21.61 & 50.46 & 5.34 & 10.02 \\
\hline & 180 & 60.47 & 5.70 & 6.46 & 5.18 & 9.91 & 20.12 & 52.34 & 4.45 & 8.78 \\
\hline & 240 & 61.62 & 5.63 & 6.39 & 4.86 & 8.21 & 14.37 & 55.99 & 4.93 & 6.59 \\
\hline & 120 & 70.58 & 6.92 & 8.10 & 6.09 & 12.45 & 26.08 & 52.59 & 6.01 & 13.09 \\
\hline \multirow[t]{3}{*}{ Against + Hand hoeing } & 180 & 73.16 & 7.16 & 7.75 & 5.71 & 11.73 & 21.82 & 55.47 & 5.53 & 10.86 \\
\hline & 240 & 75.46 & 7.09 & 7.64 & 5.23 & 10.63 & 16.29 & 59.60 & 5.48 & 7.54 \\
\hline & 120 & 65.82 & 6.13 & 7.46 & 5.66 & 12.07 & 22.00 & 50.51 & 5.50 & 10.93 \\
\hline \multirow[t]{3}{*}{ Floro } & 180 & 66.85 & 6.04 & 7.38 & 5.31 & 10.61 & 20.96 & 53.75 & 5.18 & 9.52 \\
\hline & 240 & 69.09 & 5.90 & 7.29 & 5.08 & 9.31 & 15.27 & 57.41 & 5.07 & 6.58 \\
\hline & 120 & 77.80 & 7.25 & 9.31 & 6.45 & 13.19 & 26.75 & 53.70 & 6.70 & 14.53 \\
\hline \multirow[t]{3}{*}{ Floro + Hand hoeing } & 180 & 79.33 & 7.53 & 8.51 & 6.30 & 12.32 & 23.86 & 58.49 & 5.95 & 12.41 \\
\hline & 240 & 77.17 & 7.13 & 8.41 & 6.08 & 11.22 & 17.38 & 62.21 & 5.50 & 7.87 \\
\hline & 120 & 62.25 & 5.58 & 6.50 & 5.81 & 10.19 & 20.29 & 50.11 & 5.03 & 9.53 \\
\hline \multirow[t]{2}{*}{ Stomp extra } & 180 & 63.23 & 5.40 & 6.29 & 4.82 & 9.09 & 19.32 & 52.03 & 4.80 & 8.82 \\
\hline & 240 & 64.31 & 5.37 & 6.22 & 4.39 & 7.76 & 13.87 & 51.36 & 4.66 & 6.40 \\
\hline \multirow{4}{*}{$\begin{array}{l}\text { Stomp extra }+ \text { Hand } \\
\text { hoeing }\end{array}$} & 120 & 68.08 & 6.25 & 7.73 & 5.97 & 12.23 & 23.25 & 51.99 & 5.73 & 12.39 \\
\hline & 180 & 70.71 & 6.11 & 7.54 & 5.58 & 11.23 & 21.67 & 54.49 & 5.33 & 10.30 \\
\hline & 240 & 72.27 & 6.09 & 7.42 & 5.16 & 10.37 & 15.42 & 58.60 & 5.18 & 7.09 \\
\hline & 120 & 54.86 & 5.42 & 5.99 & 4.54 & 8.80 & 15.34 & 50.04 & 4.32 & 7.65 \\
\hline \multirow[t]{3}{*}{ Hand hoeing twice } & 180 & 56.27 & 5.32 & 6.17 & 4.35 & 7.90 & 13.69 & 50.62 & 4.26 & 6.99 \\
\hline & 240 & 60.82 & 5.25 & 5.94 & 4.12 & 7.26 & 10.76 & 50.63 & 4.26 & 5.68 \\
\hline & 120 & 48.02 & 5.17 & 5.95 & 3.61 & 6.15 & 6.97 & 43.51 & 3.99 & 6.07 \\
\hline \multirow[t]{2}{*}{ Unweeded check } & 180 & 51.64 & 4.42 & 5.18 & 3.44 & 5.28 & 5.52 & 48.40 & 3.74 & 4.67 \\
\hline & 240 & 56.63 & 4.28 & 5.10 & 3.30 & 4.60 & 4.73 & 49.40 & 3.69 & 4.53 \\
\hline$\overline{\operatorname{LSD}}_{(0.05)}$ & & 2.81 & 0.36 & 0.34 & 0.29 & 0.43 & 1.10 & 3.06 & 0.34 & 0.57 \\
\hline
\end{tabular}

\section{Onion bulbs yield:}

Weed control treatments significantly increased bulb yield per faddan and its attributes in 2016/17 and 2017/18 seasons (Table 7). These parameters were significantly increased under all weed control treatments as compared to hand hoeing twice treatment or unweeded check. Floro at the rate of $750 \mathrm{~cm}^{3} / \mathrm{fad}$, in combination with hand hoeing once at 45 DAT raised the average bulb weight, marketable and total bulbs yield by about $39.69 \mathrm{~g}, 11.80$ and $10.03 \mathrm{t} / \mathrm{fad}$, respectively in 2016/17 season and these increases raised to be about $59.82 \mathrm{~g}, 12.42$ and $10.50 \mathrm{t} / \mathrm{fad}$, respectively in control treatment by Floro at rate of $750 \mathrm{~cm}^{3} /$ fad plus hand hoeing once at 45 DAT with plant density of 120 thousand plants/fad (30 plants $/ \mathrm{m}^{2}$ ) was applied, whereas the lowest values of this trait were achieved at higher planting density (240 thousand plants/fad) with untreated control in both growing seasons. 
For the influence of planting population on the onion crop and its components, data in Table 7 clearly revealed that the differences in these traits between the three plant densities were significant in both seasons. Plant density 180 thousand plants/fad gave the best marketable and total yield/fad followed by 240 thousand plants/fad then 120 thousand plants/fad, in 2016/17 and 2017/18 seasons. The impact of plant density on onion yield showed a similar trend as that number of leaves/plant, bulb diameter and plant dry weight. This result showed a clear positively correlation between early vegetative vigour, due to eliminating competition by weed growth, and plant investment towards enhancing the crop yield. Many investigation i.e., Geries et al. (2015), Ali et al. (2016 a\&b) and Abdelmasieh (2017) confirming this conclusion.

Overall, the combined interaction between weed control treatments and planting population 180 thousand plants/fad had the greatest marketable and total yield/fad of onion with the lowest culls weight compared with the other treatments including planting population 120 or 240 thousand plants/fad in the two seasons (Table 8). The highest values of this parameter (18.18 and $17.70 \mathrm{t} / \mathrm{fad})$ and (19.10 and $18.66 \mathrm{t} / \mathrm{fad})$ were registered in planting density 180 thousand plants/fad under Floro at the rate of $750 \mathrm{~cm}^{3} / \mathrm{fad}+$ hand hoeing once at 45 DAT in both 2016/17 and 2017/18 seasons, respectively. In contrast, the highest culls yield of onion (3.61 and $3.50 \mathrm{t} / \mathrm{fad}$ ) was resulted from the unweeded check under planting population 240 thousand plants/fad conditions in two seasons, respectively. The other combined interactions occupied an intermediate position. The superiority of herbicides treatments along with hand hoeing over the untreated control with 180 thousand plants/fad in bulb weight, marketable and total yield might be refer to that onion plants were exposed to low weed competition as a result of eliminating weeds, decreasing weeds which competitive with onion plants for nutritional requirements and the feasibility of maintaining yield with quality in the absence of effective weed control is strongly doubtful.

Table 7. Average bulb weight (g), marketable yield, culls yield and total yield ( $t /$ fad) as affected by some herbicides treatments and planting population in $2016 / 17$ and $2017 / 18$ seasons.

\begin{tabular}{|c|c|c|c|c|c|c|c|c|c|}
\hline \multirow[b]{2}{*}{ Character } & & \multicolumn{4}{|c|}{ 2016/17 season } & \multicolumn{4}{|c|}{ 2017/18 season } \\
\hline & & $\begin{array}{c}\text { Average } \\
\text { bulb } \\
\text { weight (g) }\end{array}$ & $\begin{array}{c}\text { Marketable } \\
\text { yield } \\
\text { (t/fad) }\end{array}$ & $\begin{array}{c}\text { Culls } \\
\text { yield } \\
\text { (t/fad) }\end{array}$ & $\begin{array}{c}\text { Total } \\
\text { yield } \\
\text { (t/fad) }\end{array}$ & \begin{tabular}{|c|} 
Average \\
bulb \\
weight (g) \\
\end{tabular} & $\begin{array}{c}\text { Marketable } \\
\text { yield } \\
\text { (t/fad) }\end{array}$ & $\begin{array}{c}\text { Culls } \\
\text { yield } \\
\text { (t/fad) }\end{array}$ & $\begin{array}{l}\text { Total } \\
\text { yield } \\
\text { (t/fad) }\end{array}$ \\
\hline \multicolumn{10}{|c|}{ Weed control treatment } \\
\hline Against & & 90.69 & 12.88 & 1.60 & 14.48 & 75.63 & 13.77 & 1.49 & 15.26 \\
\hline Against + H.H & & 105.13 & 15.62 & 0.99 & 16.61 & 93.41 & 15.36 & 1.11 & 16.47 \\
\hline Floro & & 94.20 & 13.38 & 1.38 & 14.76 & 80.75 & 14.10 & 1.36 & 15.46 \\
\hline Floro + H.H & & 112.11 & 16.43 & 0.94 & 17.37 & 106.96 & 16.07 & 0.93 & 17.00 \\
\hline Stomp extra & & 87.37 & 11.74 & 1.86 & 13.60 & 71.60 & 13.38 & 1.73 & 15.11 \\
\hline Stomp extra + H.H & & 97.44 & 14.46 & 1.06 & 15.52 & 86.34 & 14.33 & 1.24 & 15.57 \\
\hline Hand hoeing twice & & 80.84 & 11.01 & 2.12 & 13.13 & 62.61 & 12.42 & 2.11 & 14.53 \\
\hline Unweeded check & & 72.42 & 4.63 & 2.71 & 7.34 & 47.14 & 3.65 & 2.84 & 6.49 \\
\hline $\mathrm{LSD}_{(0.05)}$ & & 3.05 & 0.58 & 0.20 & 0.56 & 3.42 & 0.41 & 0.19 & 0.49 \\
\hline \multicolumn{10}{|c|}{ Planting population (thousand plants/fad) } \\
\hline 120 & & 100.24 & 11.22 & 0.99 & 12.22 & 89.04 & 11.59 & 1.10 & 12.70 \\
\hline 180 & & 95.80 & 13.72 & 1.66 & 15.39 & 79.63 & 14.06 & 1.79 & 15.85 \\
\hline 240 & & 81.55 & 12.60 & 2.10 & 14.70 & 65.31 & 12.99 & 1.91 & 14.91 \\
\hline $\mathrm{LSD}_{(0.05)}$ & & 1.94 & 0.37 & 0.17 & 0.31 & 3.46 & 0.25 & 0.16 & 0.23 \\
\hline \multicolumn{10}{|c|}{$\begin{array}{l}\text { Table 8. Average bulb weight (g), marketable yield (t/fad), culls yield (t/fad) and total yield (t/fad) as affected by the } \\
\text { interaction between some herbicides treatments and planting population in } 2016 / 17 \text { and } 2017 / 18 \text { seasons. }\end{array}$} \\
\hline \multirow[b]{2}{*}{$\begin{array}{l}\text { Weed control } \\
\text { treatment }\end{array}$} & Planting & \multicolumn{4}{|c|}{$2016 / 17$ season } & \multicolumn{4}{|c|}{$2017 / 18$ season } \\
\hline & $\begin{array}{l}\text { population } \\
\text { (thousand } \\
\text { plants /fad) }\end{array}$ & $\begin{array}{c}\text { Average } \\
\text { bulb } \\
\text { weight (g) }\end{array}$ & $\begin{array}{c}\text { Marketable } \\
\text { yield } \\
\text { (t/fad) }\end{array}$ & $\begin{array}{c}\text { Culls } \\
\text { yield } \\
\text { (t/fad) }\end{array}$ & $\begin{array}{c}\text { Total } \\
\text { yield } \\
\text { (t/fad) }\end{array}$ & $\begin{array}{c}\text { Average } \\
\text { bulb } \\
\text { weight (g) }\end{array}$ & $\begin{array}{c}\text { Marketable } \\
\text { yield } \\
\text { (t/fad) }\end{array}$ & $\begin{array}{c}\text { Culls } \\
\text { yield } \\
\text { (t/fad) }\end{array}$ & $\begin{array}{c}\text { Total } \\
\text { yield } \\
\text { (t/fad) }\end{array}$ \\
\hline \multirow{3}{*}{ Against } & 120 & 96.08 & 11.62 & 0.91 & 12.53 & 87.07 & 12.55 & 0.99 & 13.54 \\
\hline & 180 & 94.03 & 13.81 & 1.96 & 15.77 & 75.33 & 14.77 & 1.66 & 16.43 \\
\hline & 240 & 81.99 & 13.22 & 1.93 & 15.14 & 64.50 & 13.99 & 1.82 & 15.81 \\
\hline \multirow{3}{*}{ Against + H.H } & 120 & 111.66 & 13.77 & 0.51 & 14.29 & 102.93 & 13.46 & 0.83 & 14.29 \\
\hline & 180 & 109.96 & 17.07 & 1.19 & 18.26 & 101.94 & 17.34 & 1.20 & 18.54 \\
\hline & 240 & 93.96 & 16.03 & 1.26 & 17.29 & 75.37 & 15.27 & 1.31 & 16.58 \\
\hline \multirow{3}{*}{ Floro } & 120 & 99.35 & 12.21 & 0.87 & 13.08 & 94.80 & 13.07 & 0.95 & 14.02 \\
\hline & 180 & 98.17 & 14.45 & 1.46 & 15.91 & 80.22 & 15.05 & 1.62 & 16.67 \\
\hline & 240 & 85.09 & 13.47 & 1.83 & 15.30 & 67.24 & 14.17 & 1.52 & 15.69 \\
\hline \multirow{3}{*}{ Floro + H.H } & 120 & 125.82 & 14.44 & 0.66 & 15.10 & 125.79 & 14.19 & 0.69 & 14.88 \\
\hline & 180 & 114.19 & 18.18 & 0.93 & 19.10 & 107.14 & 17.70 & 0.96 & 18.66 \\
\hline & 240 & 96.32 & 16.66 & 1.23 & 17.89 & 87.94 & 16.31 & 1.14 & 17.45 \\
\hline \multirow{3}{*}{ Stomp extra } & 120 & 93.66 & 10.54 & 1.04 & 11.57 & 79.34 & 12.16 & 1.17 & 13.33 \\
\hline & 180 & 90.70 & 13.06 & 1.91 & 14.97 & 72.98 & 14.31 & 1.86 & 16.17 \\
\hline & 240 & 77.75 & 11.64 & 2.63 & 14.26 & 62.49 & 13.67 & 2.16 & 15.84 \\
\hline & 120 & 104.72 & 13.63 & 0.75 & 14.38 & 100.93 & 13.32 & 0.91 & 14.23 \\
\hline Stomp extra + H.H & 180 & 100.31 & 14.98 & 1.01 & 15.99 & 87.55 & 15.29 & 1.48 & 16.77 \\
\hline & 240 & 87.29 & 14.78 & 1.43 & 16.20 & 70.55 & 14.39 & 1.33 & 15.72 \\
\hline & 120 & 90.17 & 9.87 & 1.31 & 11.18 & 70.24 & 11.44 & 1.40 & 12.84 \\
\hline Hand hoeing twice & 180 & 83.64 & 12.66 & 2.16 & 14.82 & 62.89 & 13.22 & 2.40 & 15.61 \\
\hline & 240 & 68.73 & 10.49 & 2.90 & 13.40 & 53.25 & 12.61 & 2.54 & 15.15 \\
\hline & 120 & 80.47 & 3.73 & 1.87 & 5.59 & 51.24 & 2.55 & 1.91 & 4.46 \\
\hline Unweeded check & 180 & 75.37 & 5.62 & 2.67 & 8.29 & 48.97 & 4.82 & 3.12 & 7.94 \\
\hline & 240 & 61.43 & 4.53 & 3.61 & 8.14 & 41.21 & 3.58 & 3.50 & 7.07 \\
\hline $\operatorname{LSD}_{(0.05)}$ & & 5.29 & 0.89 & 0.35 & 0.76 & 7.36 & 0.61 & 0.36 & 0.71 \\
\hline
\end{tabular}




\section{E. Bulb quality:}

The results presented in Table 9 revealed a significant variation between the different treatments. The treatment of Floro plus hand hoeing once exhibited the better results in bulb diameter, T.S.S. and D.M. \% of onion. However, seven other herbicides treatments did not provide enough weed control and consequently, the quality of the onion had been reduced due to weed competition. Likewise, Khohlar et al., 2006 and Uygur et al., 2010 found that pendimethalin did not produce any crop response on onion plants.

Data for both seasons indicated that the onion bulb quality under the three evaluated planting population significantly differed (Table 9). The bulb diameter, T.S.S and D.M \% for plant density that had been tested were arranged as follows: $120>180>240$ thousand plants/fad in both studied seasons. The explanation of low density on these characters may be attributed to reduce competition for moisture and nutrients at low dense plants that resulted in bulbs with higher bulb weight and bigger diameter, when extra plants are overcrowded per meter, leaves are overlapped at an early stage and the benefits from light interception, on a ground area basis, are eroded. Similar results were obtained by Geries et al. (2015), Islam et al.(2015), Shock et al.(2015), Ali et al. (2016 a\&b) and Gaharwar et al. (2017).

With regard to the interaction of studies factors had significantly affected on bulb diameter in the two seasons as well as total soluble solids percentage and dry matter content in the first and second seasons, respectively (Table 10). The largest bulb with the maximum values of TSS and DM\% was produced in plots, which were sprayed with Florothand hoeing once under the lowest planting population (120 thousand plants/fad), but the lowest values were produced by unweeded check at higher planting population (240 thousand plants/fad). The results of this experiment suggest that onion crops should be kept weed-free all season long.

Table 9. Effect of different herbicides treatments and planting population on bulb quality and storability of onion bulbs for six months during 2016/17 and 2017/18 seasons.

\begin{tabular}{|c|c|c|c|c|c|c|c|c|c|c|}
\hline \multirow[b]{3}{*}{ Character } & \multicolumn{5}{|c|}{$\begin{array}{c}2016 / 17 \text { season } \\
\end{array}$} & \multicolumn{5}{|c|}{$2017 / 18$ season } \\
\hline & \multicolumn{3}{|c|}{ Bulb quality } & \multicolumn{2}{|c|}{ Storability } & \multicolumn{3}{|c|}{ Bulb quality } & \multicolumn{2}{|c|}{ Storability } \\
\hline & $\begin{array}{c}\text { Bulb } \\
\text { diameter } \\
(\mathrm{cm})\end{array}$ & $\begin{array}{c}\text { TSS } \\
\%\end{array}$ & $\begin{array}{c}\text { Dry } \\
\text { matter } \\
\%\end{array}$ & $\begin{array}{c}\text { Total } \\
\text { weight } \\
\text { loss } \%\end{array}$ & $\begin{array}{c}\text { Remained } \\
\text { bulbs } \\
\text { (t/fad) }\end{array}$ & $\begin{array}{c}\text { Bulb } \\
\text { diameter } \\
(\mathrm{cm})\end{array}$ & $\begin{array}{c}\text { TSS } \\
\%\end{array}$ & $\begin{array}{c}\text { Dry } \\
\text { matter } \\
\%\end{array}$ & $\begin{array}{c}\text { Total } \\
\text { weight } \\
\text { loss } \% \\
\end{array}$ & $\begin{array}{c}\text { Remained } \\
\text { bulbs } \\
\text { (t/fad) } \\
\end{array}$ \\
\hline \multicolumn{11}{|l|}{ Weed control treatment } \\
\hline Against & 6.30 & 14.19 & 14.76 & 28.75 & 9.14 & 6.37 & 13.73 & 15.40 & 27.84 & 9.91 \\
\hline Against + Hand hoeing & 6.91 & 15.13 & 16.18 & 22.35 & 12.08 & 7.32 & 15.30 & 17.18 & 19.94 & 12.27 \\
\hline Floro & 6.57 & 14.35 & 15.32 & 27.51 & 9.65 & 6.50 & 13.98 & 15.91 & 24.78 & 10.58 \\
\hline Floro + Hand hoeing & 7.27 & 15.66 & 16.66 & 16.86 & 13.62 & 7.42 & 16.01 & 18.05 & 14.78 & 13.67 \\
\hline Stomp extra & 5.89 & 13.62 & 14.48 & 32.74 & 7.88 & 6.13 & 13.49 & 13.18 & 31.55 & 9.12 \\
\hline Stomp extra + Hand hoeing & 6.81 & 14.82 & 15.62 & 25.46 & 10.74 & 6.74 & 14.14 & 16.26 & 22.89 & 11.03 \\
\hline Hand hoeing twice & 5.39 & 12.51 & 13.76 & 35.69 & 7.07 & 5.40 & 12.42 & 12.40 & 33.08 & 8.28 \\
\hline Unweeded check & 3.96 & 10.34 & 12.85 & 42.49 & 2.64 & 3.91 & 11.22 & 10.61 & 36.87 & 2.29 \\
\hline $\mathrm{LSD}_{(0.05)}$ & 0.28 & 0.49 & 0.94 & 4.39 & 0.68 & 0.18 & 1.06 & 0.32 & 2.13 & 0.32 \\
\hline \multicolumn{11}{|c|}{ Planting population (thousand plants/fad) } \\
\hline 120 & 7.34 & 15.00 & 15.93 & 22.47 & 8.91 & 7.10 & 14.65 & 15.82 & 20.65 & 9.37 \\
\hline 180 & 6.32 & 13.82 & 14.97 & 30.17 & 9.84 & 6.02 & 13.97 & 14.83 & 25.63 & 10.65 \\
\hline 240 & 4.75 & 12.66 & 13.97 & 34.30 & 8.54 & 5.56 & 12.74 & 13.97 & 33.12 & 8.92 \\
\hline $\mathrm{LSD}_{(0.05)}$ & 0.21 & 0.32 & 0.71 & 2.81 & 0.38 & 0.28 & 0.92 & 0.48 & 1.39 & 0.27 \\
\hline
\end{tabular}

\section{G- Economic feasibility study:}

Table 11 presents the total cost, which calculated as fixed cost (rental cost land preparation, seeding and planting, irrigation, fertilizers, weeding, harvesting, transportation and other expenses) total income and benefit/cost in relation to the different treatments. The main findings of this study show that used of Floro with hand hoeing once at 45 DAT gave the

\section{F. Storability of onion bulbs:}

Data in Table 9 revealed that the differences between weed control treatments were statistically significantly in weight 1 loss $\%$ of stored bulbs for six months in the two seasons. Thus, all studied weed control treatments along with hand hoeing treatment were significantly superior to the untreated control and weed control without hand hoeing treatments in both seasons. For this, Floro + hand hoeing once at 45 DAT gave the greatest remaining of marketable bulbs weight after storing (13.62 and $13.67 \mathrm{t} /$ fad) and significantly reduced weight loss percentage more than the unweeded check by about 60.32 and $59.91 \%$ after six months of storage in 2016/17 and 2017/18 seasons, respectively.

Regarding the influence of planting density treatment on weight loss and remained of marketable bulbs weight, data presented in Table 9 denoted that there were significant variations among different planting density treatments. After storing for six months, the lowest weight loss with the highest remaining of marketable bulbs weight was achieved by 180 thousand plants/fad following by 120 thousand plants/fad and 240 thousand plants/fad which allowed the lowest weight of remained marketable bulbs. Abdelmasieh (2017) and Abou Khadrah et al. (2017) denoted a similar results.

Data in Table 10 indicated also that bulbs weight loss percentage and remained marketable bulbs weight were insignificantly affected by combined interaction between different treatments in the first seasons, except for that in second season. The lowest values of bulbs weight loss\% were obtained by Floro followed by one hand hoeing once at 45 DAT under low planting density 120 or 180 thousand plants/fad. This could be explained in terms of increasing vegetative growth due to curbing weed stress, which ultimately lead to improve total yield and its components. (Abdelmasieh, 2017 and Abou Khadrah et al., 2017). maximum values of economic evaluation. The average of total income per fad of onion yield ranged from about 6282 L.E/fad with minimum $\mathrm{B} / \mathrm{C}$ ratio of 0.62 which was recorded with low planting density (120 thousand plants/fad) along with unweeded check treatment to about 35881 L.E/fad with maximum $\mathrm{B} / \mathrm{C}$ ratio of 3.00 when used Floro followed by hand hoeing once at 45 DAT together with planting density 
of 180 thousand plants/fad, which led to an increase in total income and net return beside benefit/cost ratio in both seasons. This was a natural result to the reduction occurred on weed growth which reflected positively on crop development consequently, rising economic return. In this concern, Sraw et al. (2016), Abdelmasieh (2017), Abou Khadrah et al. (2017) and Gaharwar et al. (2017) displayed similar findings.

Table 10. Bulb quality and storability of onion bulbs for six months as affected by the interaction between different herbicides treatments and planting population during 2016/17 and 2017/18 seasons.

\begin{tabular}{|c|c|c|c|c|c|c|c|}
\hline \multirow{3}{*}{$\begin{array}{l}\text { Weed control } \\
\text { Treatment }\end{array}$} & \multirow{3}{*}{$\begin{array}{l}\text { Planting } \\
\text { population } \\
\text { (thousand } \\
\text { plants /fad) }\end{array}$} & \multirow{2}{*}{\multicolumn{2}{|c|}{$\begin{array}{c}\text { 2016/17 season } \\
\text { Bulb quality }\end{array}$}} & \multicolumn{4}{|c|}{ 2017/18 season } \\
\hline & & & & \multicolumn{2}{|c|}{ Bulb quality } & \multicolumn{2}{|c|}{ Storability } \\
\hline & & $\begin{array}{c}\text { Bulb } \\
\text { diameter (cm) }\end{array}$ & $\begin{array}{c}\text { TSS } \\
\%\end{array}$ & $\begin{array}{c}\text { Bulb } \\
\text { diameter (cm) }\end{array}$ & $\begin{array}{c}\text { Dry } \\
\text { matter \% }\end{array}$ & $\begin{array}{l}\text { Total weight } \\
\text { loss \% }\end{array}$ & $\begin{array}{c}\text { Remained marketable } \\
\text { bulbs(t/fad) }\end{array}$ \\
\hline \multirow{4}{*}{ Against } & 120 & 7.75 & 15.30 & 7.43 & 16.29 & 21.74 & 9.83 \\
\hline & 180 & 6.41 & 14.11 & 6.22 & 15.44 & 27.10 & 10.77 \\
\hline & 240 & 4.73 & 13.16 & 5.46 & 14.48 & 34.69 & 9.13 \\
\hline & 120 & 8.55 & 16.53 & 8.13 & 18.00 & 14.68 & 11.49 \\
\hline \multirow[t]{3}{*}{ Against $+\mathrm{H} . \mathrm{H}$} & 180 & 6.74 & 14.92 & 7.20 & 17.15 & 17.49 & 14.29 \\
\hline & 240 & 5.44 & 13.95 & 6.62 & 16.40 & 27.67 & 11.04 \\
\hline & 120 & 7.85 & 15.48 & 7.52 & 16.53 & 17.96 & 10.72 \\
\hline \multirow[t]{3}{*}{ Floro } & 180 & 6.84 & 14.28 & 6.28 & 16.08 & 23.17 & 11.56 \\
\hline & 240 & 5.01 & 13.30 & 5.70 & 15.13 & 33.21 & 9.47 \\
\hline & 120 & 8.72 & 17.30 & 7.93 & 18.87 & 11.58 & 12.54 \\
\hline \multirow[t]{3}{*}{ Floro +H.H } & 180 & 7.51 & 15.32 & 7.30 & 18.13 & 15.51 & 14.96 \\
\hline & 240 & 5.59 & 14.35 & 7.05 & 17.16 & 17.26 & 13.50 \\
\hline & 120 & 6.97 & 14.75 & 7.05 & 14.69 & 24.63 & 9.17 \\
\hline \multirow[t]{3}{*}{ Stomp extra } & 180 & 6.23 & 13.79 & 5.84 & 12.85 & 32.84 & 9.61 \\
\hline & 240 & 4.48 & 12.30 & 5.50 & 12.01 & 37.19 & 8.59 \\
\hline & 120 & 8.04 & 16.15 & 7.64 & 16.96 & 17.00 & 11.06 \\
\hline \multirow[t]{3}{*}{ Stomp extra + H.H } & 180 & 7.23 & 14.40 & 6.47 & 16.33 & 21.33 & 12.03 \\
\hline & 240 & 5.16 & 13.92 & 6.10 & 15.49 & 30.35 & 10.02 \\
\hline & 120 & 6.41 & 13.57 & 6.35 & 13.82 & 26.64 & 8.39 \\
\hline \multirow[t]{3}{*}{ Hand hoeing twice } & 180 & 5.54 & 13.26 & 5.29 & 12.19 & 33.39 & 8.80 \\
\hline & 240 & 4.23 & 10.70 & 4.56 & 11.19 & 39.22 & 7.66 \\
\hline & 120 & 4.42 & 10.90 & 4.73 & 11.41 & 30.96 & 1.76 \\
\hline \multirow[t]{2}{*}{ Unweeded check } & 180 & 4.08 & 10.47 & 3.52 & 10.47 & 34.24 & 3.17 \\
\hline & 240 & 3.37 & 9.64 & 3.47 & 9.94 & 45.41 & 1.95 \\
\hline$\overline{\operatorname{LSD}}_{(0,05)}$ & & 0.60 & 0.80 & 0.36 & 0.54 & 3.29 & 0.64 \\
\hline
\end{tabular}

Table 11. Effect of some herbicides treatments and planting population on economic analysis of onion (Combined data of two years).

\begin{tabular}{|c|c|c|c|c|c|}
\hline $\begin{array}{l}\text { Weed control } \\
\text { treatment }\end{array}$ & $\begin{array}{c}\text { Planting population } \\
\text { (thousand plants/fad) }\end{array}$ & $\begin{array}{c}\text { Total Costs } \\
\text { (L.E./fad) }\end{array}$ & $\begin{array}{l}\text { Total income } \\
\text { (L.E./fad) }\end{array}$ & $\begin{array}{l}\text { Net farm return } \\
\text { (L.E./fad) }\end{array}$ & $\begin{array}{c}\text { Benefit/Cost } \\
\text { ratio }(\mathrm{B} / \mathrm{C}) \\
\end{array}$ \\
\hline \multirow{3}{*}{ Against } & 120 & 10850 & 24171 & 13321 & 2.23 \\
\hline & 180 & 10900 & 28581 & 17681 & 2.62 \\
\hline & 240 & 10950 & 27212 & 16262 & 2.49 \\
\hline & Mean & 10900 & 26655 & 15755 & 2.45 \\
\hline \multirow{3}{*}{ Against +H.H } & 120 & 12350 & 27231 & 14881 & 2.20 \\
\hline & 180 & 13900 & 34411 & 20511 & 2.48 \\
\hline & 240 & 15450 & 31301 & 15851 & 2.03 \\
\hline \multirow{4}{*}{ Floro } & Mean & 13900 & 30981 & 17081 & 2.23 \\
\hline & 120 & 10475 & 25281 & 14806 & 2.41 \\
\hline & 180 & 10525 & 29501 & 18976 & 2.80 \\
\hline & 240 & 10575 & 27641 & 17066 & 2.61 \\
\hline \multirow{2}{*}{$\longrightarrow$} & Mean & 10525 & 27474 & 16949 & 2.61 \\
\hline & 120 & 11975 & 28631 & 16656 & 2.39 \\
\hline \multirow{3}{*}{ Floro +H.H } & 180 & 12025 & 35881 & 23856 & 3.00 \\
\hline & 240 & 12075 & 32971 & 20896 & 2.73 \\
\hline & Mean & 12025 & 32494 & 20469 & 2.70 \\
\hline \multirow{3}{*}{ Stomp extra } & 120 & 10820 & 22701 & 11881 & 2.10 \\
\hline & 180 & 10870 & 27372 & 16502 & 2.52 \\
\hline & 240 & 10920 & 25312 & 14392 & 2.32 \\
\hline \multirow{4}{*}{ Stomp extra +H.H } & Mean & 10870 & 25128 & 14258 & 2.31 \\
\hline & 120 & 12320 & 26951 & 14631 & 2.19 \\
\hline & 180 & 13870 & 30271 & 16401 & 2.18 \\
\hline & 240 & 15420 & 29171 & 13751 & 1.89 \\
\hline - & Mean & 13870 & 28798 & 14928 & 2.08 \\
\hline \multirow{3}{*}{ Hand hoeing twice } & 120 & 13100 & 21311 & 8211 & 1.63 \\
\hline & 180 & 13150 & 25882 & 12732 & 1.97 \\
\hline & 240 & 13200 & 23102 & 9902 & 1.75 \\
\hline \multirow{4}{*}{ Unweeded check } & Mean & 13150 & 23432 & 10282 & 1.78 \\
\hline & 120 & 10100 & 6282 & -3818 & 0.62 \\
\hline & 180 & 10150 & 10442 & 292 & 1.03 \\
\hline & 240 & 10200 & 8113 & -2087 & 0.80 \\
\hline \multirow{4}{*}{$\begin{array}{l}\text { Planting population } \\
\text { (thousand plants/fad) }\end{array}$} & Mean & 10150 & 8279 & -1871 & 0.82 \\
\hline & 120 & 11499 & 22820 & 11321 & 1.98 \\
\hline & 180 & 11924 & 27793 & 15869 & 2.33 \\
\hline & 240 & 12349 & 25603 & 13254 & 2.08 \\
\hline
\end{tabular}

H.H: Hand hoeing 
Correlation study:

Correlation coefficient estimations were done for some characters studied over the two seasons (Table 12). Significant positive correlations were recorded among dry weight of broadleaf, grassy and total weeds and culls yield/fad. The values of the correlation coefficient between dry weight of broadleaf, grassy and total weeds at 90 DAT and each of average bulb weight, marketable yield/fad, total bulbs yield and remaining of marketable bulbs weight after storing for six months were negative and significant, while the correlations between bulb weight and each of marketable yield/fad, total bulbs yield and remaining of marketable bulbs weight after storing for six months were significant and positive. Also, correlation analysis revealed that the yield increases as a result of decreased weed competition which positively contributed to the increases in growth characters and yield components. The correlation between dry weight of grasses, broadleaved, total species and onion yield was statistically significant and negative. The correlation among marketable yield/fad and each of total bulbs yield and remaining of marketable bulbs weight after storing for six months was significant and positive. While, Correlation among marketable yield/fad and culls yield/fad was significant and negative. Hence, weed control plays a major role in increasing onion productivity and its quality, however these features are conditional; if applied at the suitable time, rate and stage of weed growth.

Table 12. Correlation coefficient between some studied characteristics and onion yield and its components, (combined data of 2016/17 and 2017/18 seasons).

\begin{tabular}{|c|c|c|c|c|c|c|c|}
\hline Studied characteristics & $\begin{array}{c}\text { Dry weight } \\
\text { of grassy } \\
\text { weeds }\left(\mathrm{g} / \mathrm{m}^{2}\right)\end{array}$ & $\begin{array}{c}\text { Dry weight } \\
\text { of total } \\
\text { weeds }\left(\mathrm{g} / \mathrm{m}^{2}\right)\end{array}$ & $\begin{array}{c}\text { Bulb } \\
\text { weight } \\
\text { (g) }\end{array}$ & $\begin{array}{c}\text { Marketable } \\
\text { yield } \\
\text { (t/fad) }\end{array}$ & $\begin{array}{c}\text { Culls } \\
\text { yield } \\
\text { (t/fad) }\end{array}$ & $\begin{array}{l}\text { Total } \\
\text { yield } \\
\text { (t/fad) }\end{array}$ & $\begin{array}{c}\text { Remained } \\
\text { marketable } \\
\text { bulbs (t/fad) }\end{array}$ \\
\hline Dry weight of broad-leaved weeds $\left(\mathrm{g} / \mathrm{m}^{2}\right)$ & $0.98^{m-1}$ & $0.99^{\cdots \prime \prime}$ & $-0.56^{\cdots \cdots}$ & $-0.91^{m *}$ & $0.54^{\cdots \cdots}$ & $-0.90^{\cdots \cdots}$ & $-0.83^{\cdots \cdots}$ \\
\hline Dry weight of grassy weeds $\left(\mathrm{g} / \mathrm{m}^{2}\right)$ & & $0.98^{n-}$ & $-0.52^{\cdots \cdots}$ & $-0.90^{\cdots-}$ & $0.51^{*-}$ & $-0.89^{\cdots+}$ & $-0.81^{* \pi}$ \\
\hline Dry weight of total weeds $\left(\mathrm{g} / \mathrm{m}^{2}\right)$ & & & $-0.55^{\infty \cdots}$ & $-0.91^{* *}$ & $0.54^{-\cdots}$ & $-0.89^{\ldots-\infty}$ & $-0.83^{\cdots+}$ \\
\hline Bulb weight $(\mathrm{g})$ & & & & $0.66^{* \infty}$ & $-0.86^{n-\pi}$ & $0.55^{\infty-}$ & $0.83^{n-}$ \\
\hline Marketable yield (t/fad) & & & & & $-0.62^{\cdots \cdots}$ & $0.98^{\cdots+}$ & $0.95^{n-}$ \\
\hline Culls yield $(\mathrm{t} / \mathrm{fad})$ & & & & & & $-0.47^{n-\infty}$ & $-0.75^{-\infty}$ \\
\hline Total yield ( $\mathrm{t} / \mathrm{fad})$ & & & & & & & $0.89^{\cdots+}$ \\
\hline
\end{tabular}

** Correlation is highly significant at $1 \%$ probability.

\section{CONCLUSION}

In conclusion, integration of herbicides with manual weeding and the proper plant density exhibited excellent weed control efficiency coupled with good outcome on yield productivity. From the results of the present study it can be concluded that the use of integration between Floro EC 24\% $\left(750 \mathrm{~cm}^{3} / \mathrm{fad} ; 21\right.$ days after transplanting) in combination with hand hoeing once (45 DAT) plus plant density of 180 thousand plants/fad performed comparatively well than any other treatment at all levels, and is strongly suggested to the onion's farmers of this area.

\section{REFERENCES}

Abdelmasieh, W.K.L. (2017). Onion productivity and quality as influenced by planting date, method and density. Ph.D. Thesis, Fac. Agric., Kafrelsheikh Univ., Egypt.

Abou Khadrah, S.H.; A. A. El-Sayed; L. S. M. Geries and W.K.L. Abdelmasieh (2017). Response of onion yield and quality to different planting date, methods and density. Egypt.J.Agron., 39(2):203 - 219.

Ali, L.; M.W. Hassan; M. Jamil; J. Iqbal; M.S. Yaqub; M. Akram; I. Ahmed and A. Hussain (2016 a). Effect of nursery bulb size and planting density on Thirps population, plant height and yield of onion (Phulkara variety) in Bahawalpur, Pakistan. Pak. J. Life Soc. Sci., 14(2): 96-103.

Ali, M.; A. Rab; J. Ali; H. Ahmad; S. Hayat; K. Wali; A. Rashid; W. Ahmad and H. Muhammad (2016 b). Influence of transplanting dates and population densities on the growth and yield of onion. Pure Appl. Biol., 5(2):345-354.
Bardisi, Samar A.; E.A. El-Ghamriny; A.A. Gad and Nawar, A.S. Dalia (2013). Bulbing ratio and dry weight of onion plant grown in sandy soil as affected by planting date, planting distance and potassium fertilizer rate. Zagazig J. Agric. Res., 40(3): 399-410.

Chandrika, V., Reddy, D. Srinivasulu, K. Sagar, G. Reddy and Prabhakara (2009). Influence of graded levels of nutrients, time of $\mathrm{N}$ application and weed management practices on weed dynamics, yield attributes and bulb yield of onion (Allium cepa L.). Indian J. of Weed Sci., 41(1\&2): 80-89.

Channapagoudar, B.B. and N. R. Biradar (2007). Physiological studies on weed control efficiency in direct sown onion. Karnataka J. Agri. Sci., 20(2): 375-76.

Cimmyt (1988). "From Agronomic Data to Farmer Recommendation: An Economic Work Book" D.F: pp. 31- 33.

Elian, H. M. A.; G. A. Sary; A. Roshdy; N. Kh. ElGizawy; M. R. Moshtohry and S. D. M. Eid (2016). Effect of weed control and $\mathrm{N}, \mathrm{K}$ fertilizers on productivity of onion (Allium cepa, L.) and associated weeds under new land soils. Am-Euras. J. Agric. \& Environ. Sci., 16 (2): 348-356.

Frans, R.E. and R. Talbet (1977). Design of field experminent and the measurement and analysis of plant response. Res. Methods in Weed Sci. Soc. Field. South. USA, Aburn, Alabama.

Gaharwar, A. M.; N. Patil and D. Ughade (2017). Effect of integrated weed management on growth, yield and economic returns on onion (Allium cepa, L.). Asian J. Hort., 12(2): 193-197. 
Geries, L. S. M.; E. A. Moursi and A.M.A. Abo - Dahab (2015). Effect of irrigation levels, cultivation methods and plant densities on productivity, quality of onion crop and some water relations in heavy clay soils. J. Soil Sci. and Agric. Eng., Mansoura Univ., 6(12): 1467 - 1495 .

Gomez, K. A. and A. A. Gomez (1984). Statistical procedures for agricultural research (Second Ed.) John Willey and Sons, New York, pp: 680.

Hussain, Z.; K. B. Marwat; A. Shah; S. A. Arifullah and N. M. Khan (2008). Evaluation of different herbicides for weed control in onion. Sarbad, J.Agric., 24 (3): 453-456.

Islam, M. R.; A. Mukherjee; K.G. Quddus; P. K. Sardar and M. Hossain (2015). Effect of spacing and fertilizer on the growth and yield of onion. Int. J. Sci. Tech. Res., 4(10): $308-312$.

Jackson, M. I. (1973). Soil Chemical Analysis. Prentice Hall of India Private, LTD New Delhi.

Jilani, M. S.; M. Ramzan and K.Waseem (2007). Impact of weed management practices on growth and yield of some local genotypes of onion. Pak. J. weed Sci. Res., 13 (3-4) 191-198.

Kalhapure, A. H; B. T. Shete and D. Madhukar (2014). Weed management in onion by pre-planting and post-emergence herbicides for seed production. Indian J. Weed Sci., 46(2):142-145.

Kalhapure, A. H. and B. T. Shete (2012). Integrated weed management in onion.Indian J. Weed Sci., 44(2): 88-91.

Kalhapure, A.H., Shete, B.T. and P.S. Bodake (2013). Integarted weed management in onion (Allium cepa, L.). Indian J. Agron., 58 (3): 408-411.

Khokhar, K. M., T. Mahmood, M. Shakeel and M. F. Chaudhry (2006). Evaluation of integrated weed management practices for onion in Pakistan. Crop Protection, 25: 968-972.
Klute, A. C. (1986). Water retention: Laboratory Methods, In:A. Koute(ed.), Methods of Soil Analysis, part 12nd(ed.)Agron Monogr.9, ASA, Madison, W, USA, pp.635-660.

Kumara, S., S. Das, R. Kumar and Kavita (2018). Herbicide mixture for enhancing weed control efficiency and yield of onion (Allium cepa, L.) Int.J.Curr.Microbiol.App. Sci., Special Issue-7: 2710-2714

Ramalingam, S. P.; C. Chinnagounder; M. Perumal and M. A. Palanisamy (2017). Evaluation of new formulation of oxyfluorfen $(23.5 \%$ EC) for weed control efficacy and bulb yield in onion. American Journal of Plant Sciences, 4: 890-895.

Shock, C.C.; E.B.G. Feibert; A. Riveira and L. D. Saunders (2015). Response of onion yield, grade, and financial return to plant population and irrigation system. Hort Science, 50(9): 1312-1318.

Sraw, P. K.; B. Kaur, A. Kaur and K. Singh (2016). Efficacy of different herbicides for controlling weeds in onion. Journal of Crop and Weed, 12(1):125-128.

Steel, R. G. D. and Jour. H. Torrie (1980). Principles and Procedures of Statistics. MC. Graw Hill Book Company Inc. New York, 481 pp.

Swaify, N. G. (2009). Studies on weed control in onion, Ph.D. Theses, Fac. Agric., Al-Azhar Univ., Cairo, Egypt.

Uygur, S., R. Gurbuz and F.N. Uygur (2010). Weeds on onion fields and effects of some herbicides on weeds in Cukurova region, Turkey. African J. Biotechnol. 9:7037-7042.

Walle, T., N. Dechassa and K. Tsadik (2018). Yield and yield components of onion (Allium cepa, var. cepa) cultivars as influenced by population density at Bir Sheleko, North-Western Ethiopia. Acad. Res. J. Agri. Sci. Res., 6(3):172-192.

\section{كفاية طرق مكافحة الحشائ وكثافة الزراعة والجدوي الأقتصادية علي مكافحة الحشائ وأنتاجية محصول البصل

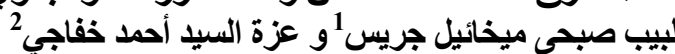

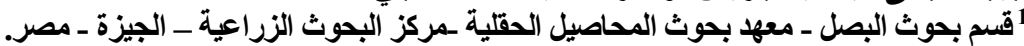

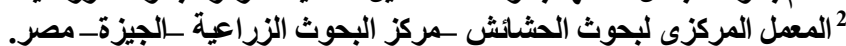

أجريت تجربة حقلية بحطة البحوث الزر اعية بسخا - محافظة كفر الثيخ - خلال الموسمين 2017/2016 مو و 2017/

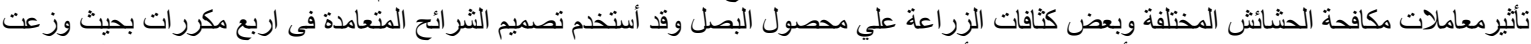

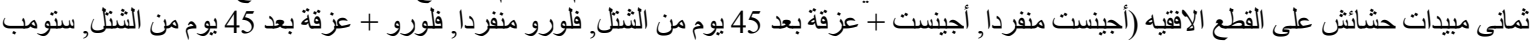

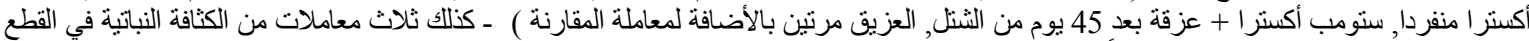

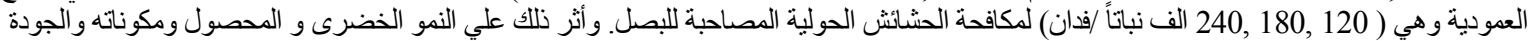

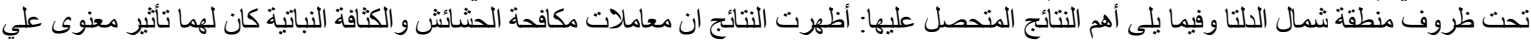

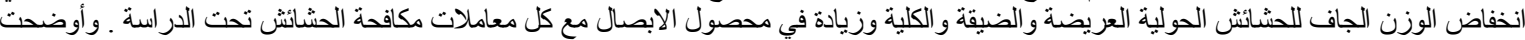

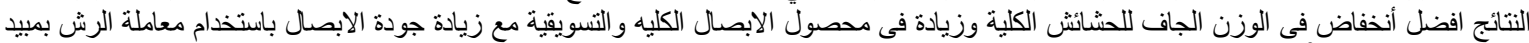

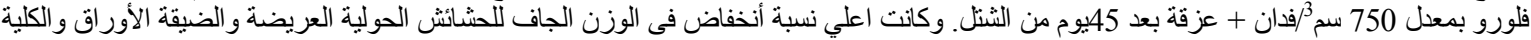

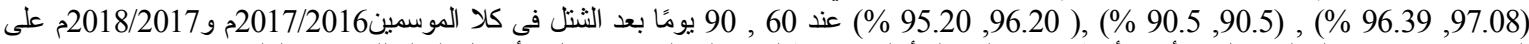

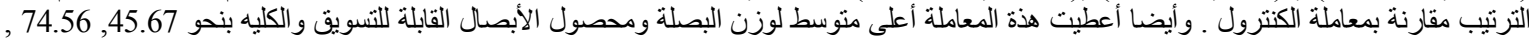

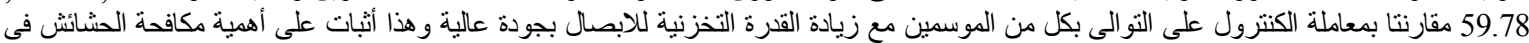

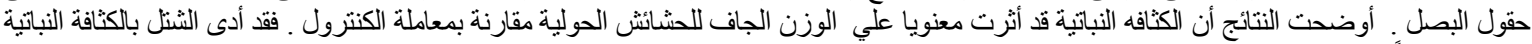

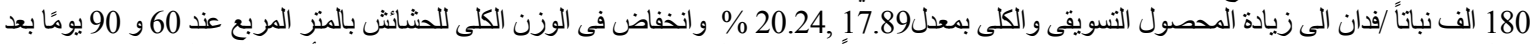

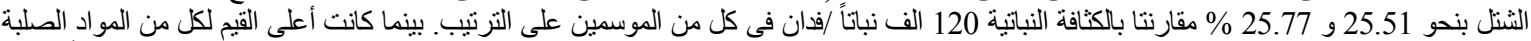

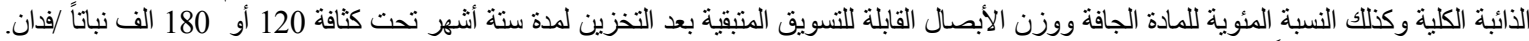

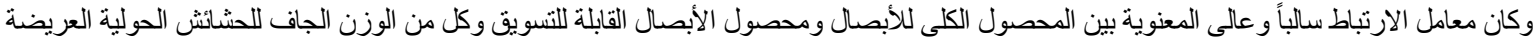

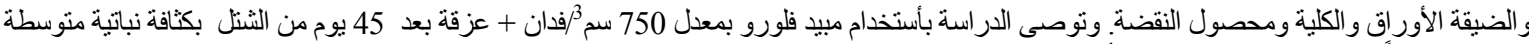
(180 الف نباتًاً /فدان) وذللك للحصول على أعلى محصول بوحدة المساحه بجودة عاليه وزيادة العائد الاقتصادى مع زيادة القدرة التخزنيه للابصال. 